\title{
Blumenberg ou les intermittences de la conscience
}

\section{Marion Schumm}

\section{OpenEdition}

\section{Journals}

Édition électronique

URL : http://journals.openedition.org/alter/375

DOI : 10.4000/alter.375

ISSN : 2558-7927

\section{Éditeur :}

Association ALTER, Archives Husserl (CNRS-UMR 8547)

\section{Édition imprimée}

Date de publication : 1 novembre 2015

Pagination : 76-97

ISBN : 978-2-9550449-1-9

ISSN : $1249-8947$

\section{Référence électronique}

Marion Schumm, «Blumenberg ou les intermittences de la conscience », Alter [En ligne], 23 | 2015, mis en ligne le 01 décembre 2017, consulté le 05 mai 2019. URL : http://journals.openedition.org/alter/375 ; DOI : 10.4000/alter.375 


\title{
BLUMENBERG OU LES INTERMITTENCES DE LA CONSCIENCE
}

\begin{abstract}
Marion Schumm
Nous ne saurons jamais - selon le célèbre Ignorabimus de Du Bois-Raymond - ce qu'est la conscience ; mais cela ne nous dispense pas de l'impératif théorique de la décrire adéquatement dans ses réalisations, même si cette description nous agace par son caractère inextricable ${ }^{1}$.
\end{abstract}

\section{Introduction}

Dans ses phénomènes les plus habituels et quotidiens, la vie consciente est traversée d'interruptions, elle se diffracte en ses manifestations, connait des lacunes et des ruptures. Comment comprendre et décrire une conscience tantôt distraite, tantôt attentive, donc les vécus passent insensiblement ou brusquement de l'actualité à la latence et inversement, une conscience qui plonge dans le sommeil, qui somnole, et se réveille, qui s'absente à elle-même et se retrouve ? Depuis sa lecture critique de Husserl, Hans Blumenberg élabore les linéaments d'une phénoménologie hétérodoxe de la conscience qui entreprend d'en penser l'unité depuis les manifestations de son intermittence.

La question de l'identité à soi-même ouvre de vastes débats, dont les contours conceptuels et historiques ne sont pas aisément déterminables (et Blumenberg lui-même ne retrace que partiellement les horizons et enjeux théoriques dans lesquels son questionnement peut

\footnotetext{
${ }^{1}$ H. Blumenberg, Description de l'homme, trad. D. Trierweiler, Paris, Cerf, « Passages », 2007 (désormais DH), p. 152.
} 
$s^{\prime}$ inscrire $\left.{ }^{2}\right)$, sinon peut-être en négatif. L'identité dont il sera question ici n'est pas à entendre comme l'unité formelle de l'ego : elle est en revanche problématisée depuis les difficultés et apories que rencontre la phénoménologie transcendantale de Husserl. Il ne s'agit donc pas, pour Blumenberg, de réinvestir la possibilité d'une philosophie « égologique ", que celle-ci se donne pour tâche de fonder l'identité du sujet ou d'élucider le sens de la corrélation intentionnelle. Il n'est pas non plus, du moins directement, question de l'identité au sens de la biographie personnelle et de son éventuelle structure narrative. Plutôt faudrait-il affirmer que Blumenberg, en amont de la question de l'identité personnelle, s'intéresse à la capacité élémentaire de s'identifier comme un et le même, de s'attribuer ou s'approprier spon-

tanément ses vécus actuels et passés, capacité qui repose sur des opérations implicites qu'il s'agit de décrire dans le cadre d'une phénoménologie anthropologisée. C'est en effet d'après lui en raison de l'interdit de l'anthropologie qui détermine la phénoménologie dès son principe qu'Husserl ne peut traiter de façon satisfaisante de ce problème. En donnant pour but à la phénoménologie de s'attacher à la conscience pure - dont la conscience humaine ne serait donc qu'une instanciation contingente - Husserl s'oblige à un partage entre les traits purs, transcendantaux et les traits contingents, seulement " psychologiques » de la conscience qui lui interdit en dernière instance de considérer pour elles-mêmes les opérations de l'identité.

Il nous faut donc tout de suite préciser le statut ou l'enjeu de cette critique : elle ne vise pas tant à récuser la phénoménologie en raison de ses contradictions ou apories, quitte à méconnaître les inflexions qu'Husserl lui a fait prendre au cours de son œuvre, que de se servir de ses problèmes et difficultés pour ouvrir à un tout autre projet, résolument non transcendantal, qui rompt avec les ambitions originaires et toujours efficaces de la phénoménologie ${ }^{3}$.

\footnotetext{
${ }^{2}$ On peut cependant penser que sa pensée pourrait gagner à être confrontée à celles de MerleauPonty, Ricœur et Descombes sur la question de l'identité, ce qui dépasse ce que nous nous proposons de faire dans cette contribution.

${ }^{3}$ Sur le rapport critique de Blumenberg à la phénoménologie, voir notamment J.-C. Monod, " "L'interdit anthropologique" chez Husserl et Heidegger et sa transgression par Blumenberg ", Revue germanique internationale, $\mathrm{n}^{\circ}$ 10, en ligne, 2009, et A. Schnell, «Le projet blumenbergien d'une anthropologie phénoménologique ", in Hans Blumenberg, anthropologie philosophique, coordonné par D. Trierweiler, Paris, PUF, «Débats philosophiques », 2010. Nous nous permettons en outre de renvoyer à notre article: "Blumenberg et la phénoménologie ", paru dans «Implications philosophiques »: http://www.implications-philosophiques.org/actualite/une/ blumenberg-et-la-phenomenologie/
} 
Si nous souhaitons nous intéresser dans notre contribution aux intermittences de la conscience, ce n'est pas parce que celles-ci permettraient, selon Blumenberg, de venir combler un déficit anthropologique de la phénoménologie, au sens d'un chapitre manquant que l'on pourrait simplement lui adjoindre. Voilà qui ne pourrait que manquer le sens même des pensées respectives de Husserl et de Blumenberg. La phénoménologie de la conscience intermittente que l'on peut lire chez Blumenberg n'est pas un ensemble de développements concrets qui viendraient enrichir une phénoménologie trop aride ou éloignée de l'expérience quotidienne.

En choisissant de partir des phénomènes de fragmentation, de latence, d'intermittence de la conscience, Blumenberg adopte un autre regard sur les intentions fondatrices de la phénoménologie, met en question ses métaphores directrices pour mieux poser la conscience comme problème : ce sont les difficultés de la phénoménologie à traiter des difficultés que connaît (au double sens du terme) la conscience qui mettent sur la piste d'une description plus satisfaisante de ces dernières. L'absence momentanée à soi, le souvenir et ses lacunes, l'opacité de la conscience sont des défis autant pour la phénoménologie de la conscience que pour la conscience elle-même : elles mettent en jeu ses opérations intentionnelles et l'identité à travers le temps, mais constituent aussi, c'est là une des thèses de Blumenberg, les moyens de son identité.

Nous nous attacherons donc à présenter différentes formes $\mathrm{d}^{\prime}$ « intermittence », terme englobant un grand nombre de phénomènes, parmi lesquels nous distinguerons entre ceux de la discontinuité et ceux de la rupture. Les interruptions de l'intentionnalité, le souvenir et l'oubli, le sommeil et l'éveil sont au cœur de la description de la conscience par Blumenberg, qui va de pair avec une critique des métaphoriques directrices de Husserl : celles du flux et du pôle. Nous montrerons ensuite dans quelle mesure c'est l'intermittence même qui permet de reposer le problème de l'identité à soi de la conscience : celle-ci n'est pas à penser au sein des vécus ou au-delà d'eux, mais bien dans la réparation et la reprise de sa discontinuité.

\section{Discontinuités et intermittences de la conscience : la nécessité d'une anthropologie phénoménologique}

Conscience pure versus conscience humaine 
La conscience humaine ne saurait être, pour Husserl, le thème privilégié de la phénoménologie. Bien au contraire, c'est la conscience pure qui doit être interrogée et élucidée, dans un rejet constitutif de toute forme d'anthropologie ${ }^{4}$. C'est contre ce geste que Blumenberg pose le problème de la possibilité $\mathrm{d}^{\prime} u$ ne anthropologie phénoménologique ${ }^{5}$. Sa lecture critique de Husserl montre dans quelle mesure la conscience qui fait l'objet des investigations phénoménologiques est un produit de la phénoménologie elle-même : c'est par les procédures d'une ascèse qui la purifie de ses déterminations anthropologiques que la conscience pure est préparée, et coïncide avec le sujet phénoménologique.

C'est sur les implications problématiques de cette démarche que s'arrête Blumenberg, qui montre dans quelle mesure Husserl, dans sa recherche des traits purs de la conscience, lui attribue en réalité des traits faussement essentiels : ils sont nécessaires non à la conscience mais bien à la méthode phénoménologique. Court-circuitant la distinction entre essence et contingence qui se situe au fondement des distinctions husserliennes, Blumenberg entend affirmer que la phénoménologie doit justement s'intéresser aux traits contingents de la conscience, à ceux dont on ne peut précisément pas dire qu'une conscience en soi serait impensable sans eux ${ }^{6}$.

Dans cette perspective, la figure de la conscience « pure » ou plutôt purifiée vaut désormais comme une épreuve contrefactuelle, qui amène à s'interroger sur les discontinuités, les hiatus ou les interruptions, qui ne peuvent apparaître en rapport avec ses traits essentiels que comme des dérangements contingents.

Mais peut-on véritablement affirmer que Husserl méconnaîtrait l'incomplétude de la conscience ou ses phénomènes limites? Que la conscience ne puisse jamais saisir d'objet dans son entièreté, que la perception ne se réalise que par esquisses, cela Husserl l'a lui-même établi et longuement analysé ${ }^{7}$. Blumenberg montre qu'au delà de cette prise en compte de l'incomplétude fondamentale de l'intentionnalité, l'attachement de Husserl à un idéal de la conscience l'empêche de penser conséquemment sa discontinuité.

\footnotetext{
${ }^{4}$ Voir par exemple H. Blumenberg, Zu den Sachen und zurück, Francfort-sur-le-Main, Suhrkamp, 2007 (désormais ZSZ), p. 175. Voir également DH, première partie, et J.-C. Monod, " "L'interdit anthropologique" chez Husserl et Heidegger et sa transgression par Blumenberg ", art. cit.

${ }^{5}$ Principalement dans DH, ZSZ et Theorie der Lebenswelt, Francfort-sur-le-Main, Suhrkamp, 2010 (désormais TL).

${ }^{6}$ Voir ZSZ, p. 208.

${ }^{7}$ Voir Idées directrices pour une phénoménologie pure et une philosophie phénoménologique I (désormais Ideen I), § 44 .
} 


\section{L'intentionnalité fragmentaire}

Le constat par lequel Blumenberg enclenche sa critique de la conception husserlienne de l'intentionnalité pourrait sembler trivial, voire manquant radicalement le sens même de la réduction : la vie quotidienne ne peut faire autrement que se satisfaire de "fragments $d$ 'intuition $»^{8}$ : l'intuition pure est elle véritablement le «summum bonum» de tous les processus de la conscience, la réalisation de l'intentionnalité ?

C'est bien un motif anthropologique qui fait naître ce doute: si l'intuition était la réalité pure et définitive de la conscience, alors l'être humain en serait en large part exclu, quoi qu'il puisse accomplir avec l'aide de la phénoménologie; car il ne l'atteint que de manière épisodique, intermédiaire, éphémère, transitoire ${ }^{9}$.

Non seulement, comme l'affirmait Husserl, l'intuition est non clôturable, nécessairement incomplète, mais surtout sa continuité ne constitue pas le modèle ou la quintessence de ses vécus qui doivent être compris comme fragmentaires et discrets - au sens mathématique du terme. Ils s'interrompent ou sont interrompus par d'autres vécus perceptifs, mais aussi par des opérations symboliques ou conceptuelles, qui empêchent de caractériser l'intentionnalité comme continuité. En conséquence, il faut mettre fin, pour décrire de façon satisfaisante les vécus de la conscience, à la prépondérance du schéma de la mélodie ou de l'objet perçu dont on fait le tour, car ils laissent entendre que l'intentionnalité serait essentiellement ce processus où les impressions de la même chose s'ajoutent les unes aux autres de façon continue et homogène ${ }^{10}$. Contre les implications de la métaphorique du flux (voir plus bas), Blumenberg met l'accent sur les hiatus de la perception ${ }^{11}$.

Blumenberg en revient donc à ce que Husserl désignait comme la contradiction ou tension fondamentale de la perception extérieure, selon laquelle l'intentionnalité de la perception viserait une donation intégrale de l'objet transcendant, qui ne peut pourtant jamais être saisi dans son intégralité ${ }^{12}$. Voilà qui pointe pour Blumenberg le paradoxe moteur de la phénoménologie : soit la conscience est conscience

\footnotetext{
${ }^{8}$ Voir TL, p. 193.

${ }^{9}$ ZSZ, p. 118 sq. Nous traduisons.

${ }^{10}$ Voir ZSZ, p. 100.

${ }^{11}$ Voir ZSZ, p. 235.

12 Voir E. Husserl, Husserliana XI, Analysen zur passiven Synthesis. Aus Vorlesungs- und Forschungsmanuskripten, 1918-1926, Margot Fleischer (éd.), La Haye, Martinus Nijhoff, 1966 (Hua $\mathrm{XI})$, Introduction $\S 1$.
} 
d'un sujet transcendantal qui ne connaît pas de limitation en soi et qui pourrait donc, potentiellement au moins, intégrer l'ensemble des esquisses infinies dans une donation complète. Soit il existe des ruptures consubstantielles à ses opérations, et dans ce cas, il faut se résoudre à considérer le sujet comme humain, d'un point de vue donc anthropologique.

De plus, si l'intentionnalité de la conscience n'implique pas sa continuité, elle ne garantit pas non plus son identité :

Il n'y a de prime abord pas de connexion nécessaire entre l'intentionnalité de la conscience et son identité au sens d'un rapport à soi tout au long d'une vie. [...] Il serait envisageable que l'intentionnalité de la conscience s'épuise à chaque fois qu'elle abandonne son objet. Les phases de la conscience ne sauraient alors rien l'une de l'autre; il n'y en aurait pour chacune aucune autre, et l'unité de la conscience ne serait qu'une manière de décrire les choses du point de vue d'un observateur fictif [...]. Que signifie cet état de chose qui est du moins pensable? Pour le moins, que la phénoménologie doit concéder que l'identité de la conscience n'est pas la condition de possibilité de son intentionnalitéé ${ }^{13}$.

\section{L'identité discrète}

La vie consciente n'est en effet pas seulement soumise à des interruptions dans ses vécus intentionnels, mais également à des ébranlements plus radicaux. Il ne s'agit plus seulement ici de pointer les inflexions factuelles des vécus intentionnels, mais bien d'interroger l'identité de la conscience à travers le temps. Car celle-ci est constamment à l'épreuve d'expériences aussi quotidiennes que troublantes, dont la phénoménologie ne peut faire l'économie dans ses descriptions. Or Husserl a bien lui-même thématisé les phénomènes du sommeil, de l'oubli ou de la mort ${ }^{14}$. Mais selon l'analyse de Blumenberg, en les comprenant comme des formes empiriques de l'autoconstitution et de l'auto-individuation de la "monade ", il a manqué leur statut et les questions qui se posent à leur propos ${ }^{15}$.

\footnotetext{
${ }^{13}$ ZSZ, p. 137 sq. Nous traduisons.

${ }^{14}$ Voir à ce propos par exemple J. Dodd, «Death and Time in Husserl's C-Manuscripts » et S. Geniusas, "On Birth, Death, and Sleep in Husserl's Late Manuscripts on Time », in On Time new contributions to the husserlian phenomenology of time, Phaenomenologica 197, D. Lohmar et I. Yamaguchi (éd.), Dordrecht/Heidelberg/Londres/New York, Springer, p. 51-70 et p.71-90.

${ }^{15}$ Voir Méditations cartésiennes $\$ 33$ à propos du concept de «monade ». Sur le problème des relations entre ego transcendantal et ego psychologique, voir J. Benoist, Autour de Husserl, L'ego et la raison, Paris, Vrin, 1994. Sur ce terrain, l'analyse de Blumenberg est bien évidemment redevable de la réinterprétation par Heidegger de la phénoménologie, quoiqu'elle n'en emprunte pas les voies, ni se s'accorde avec elle sur les enjeux de ces problèmes.
} 
Anthropologies philosophiques 


\section{Se souvenir, oublier}

Si la rétention, «queue de comète » des visées intentionnelles ${ }^{16}$, appartient nécessairement à l'essence de toute conscience, il n'en va pas de même pour le souvenir. Blumenberg souligne dans quelle mesure les thématisations de Husserl tendent à faire passer le souvenir du côté de la nécessité de la conscience quand il en est non seulement un phénomène contingent ou " accessoire ${ }^{17}$ comme le dit Blumenberg, mais de plus un phénomène révélateur de sa contingence. Ainsi, par exemple, la désignation de la rétention comme «souvenir récent» nous induit en erreur en laissant penser que le second pourrait n'être que la prolongation de la première. La rétention est la condition de possibilité de la conscience du temps, ellemême condition de l'intentionnalité. Le souvenir en revanche appartient à cette catégorie de phénomènes contingents pour la conscience, qui la caractérisent factuellement et ne peuvent donc s'intégrer sans reste à une analyse transcendantale, ni être récupérés a posteriori comme une simple détermination secondaire. Le souvenir, tout comme l'attente, ne peut appartenir qu'à une conscience principiellement limitée, pour laquelle la rétention et la protention s'interrompent de facto $^{18}$. Là encore, la "conscience pure» vaut pour Blumenberg comme figure contrefactuelle : puisqu'il est tout à fait possible d'imaginer une conscience sans souvenir, celui-ci n'appartient pas nécessairement à l'essence de toute conscience et ce dont il faut rendre compte n'est pas seulement la constitution du souvenir ou ses différentes modalités, mais bien le fait même d'une conscience qui se souvient, la nécessité non transcendantale du souvenir pour la conscience.

Cette interprétation peut sembler injuste ou un peu rapide, en ce sens qu'elle ne rend pas compte en détail des développements que Husserl a consacrés au souvenir et ses différentes modalités. Ce qui intéresse Blumenberg, une fois de plus, n'est pas tant de reconstruire la cohérence et la richesse des distinctions conceptuelles du fondateur de la phénoménologie, mais plutôt de saisir ses difficultés descriptives comme autant de signes indiquant les voies alternatives pour une phénoménologie anthropologisée. Ainsi dans Chose et espace, par exemple, Husserl donne au souvenir l'aspect d'une prospection spa-

\footnotetext{
${ }^{16}$ Voir E. Husserl, Husserliana X, Zur Phänomenologie des inneren Zeitbewussteeins (1893-1917), R. Boehm (éd.), La Haye, Martinus Nijhoff, 1969.

${ }_{17}$ ZSZ, p. 207 sq.

${ }^{18}$ Voir ZSZ, p. 215-222.
} 
tiale $^{19}$. Or il s'agit là d'une analogie trompeuse, montre Blumenberg. Le schéma du « pas à pas » ne vaut pas pour le souvenir. L'idée selon laquelle l'ensemble du passé continuerait de subsister et pourrait être exploré et invoqué à nouveau est une illusion. Le processus du «se souvenir » ne se déroule jamais sous la forme d'un " rebrousser chemin » continu et homogène, mais par des raccourcis discontinus, qui font coïncider l'identité du je souvenant et du je souvenu passéz ${ }^{20}$ sans pour autant nécessairement pouvoir assurer, d'un point de vue transcendantal, leur identité21.

Blumenberg comprendra la faculté ou la capacité de se souvenir comme la réponse à un embarras, une difficulté, un risque, dont il considère qu'ils ne sont pas correctement pris en compte par Husserl. Une conscience capable de se souvenir est une conscience qui doit se souvenir. Cette perspective entraîne à réinscrire le souvenir dans une description anthropologique, autant qu'à s'intéresser aux embarras auxquels il répond et qu'il provoque à son tour, en tant qu'il recèle toujours l'ambigüité de la coïncidence et de la décoïncidence du sujet avec lui-même.

\section{S'endormir, s'éveiller}

Face aux phénomènes du souvenir, mais surtout du sommeil et du réveil, la phénoménologie est contrainte à un passage à la limite : la conscience bien sûr, ne peut faire l'expérience de sa propre abolition (que celle-ci soit temporaire ou non)22. Cela implique-t-il que la phénoménologie ne pourrait ou ne devrait se préoccuper du sommeil ou des phénomènes "limites » en général ${ }^{23}$ ? Mais une phénoménologie de la vie consciente ne requiert-elle pas que l'on entreprenne de rendre compte de ce qui la traverse le plus quotidiennement, y compris si les descriptions qui peuvent en résulter ne se donnent que comme descriptions de limites?

Au début de Höhlenausgänge ${ }^{24}$, Blumenberg se réfère au début de La recherche du temps perdu de Proust et affirme : " Aucune conscience

\footnotetext{
${ }^{19}$ Voir ZSZ p. 216 sq.

${ }^{20}$ Voir ZSZ, p. 218 sq.

${ }^{21}$ Voir H. Blumenberg, Quellen, Ströme, Eisberge, U. von Bülow et D. Krische (éd.), Francfort-surle-Main, Suhrkamp, 2012 (désormais QSE), p. 121.

${ }^{22}$ Voir ZSZ, p. 265. Ainsi que H. Blumenberg, Lebenszeit und Weltzeit, Francfort-sur-le-Main, Suhrkamp, 1986 (désormais LZWZ).

${ }^{23} \mathrm{Il}$ serait sur ce point intéressant de comparer ces analyses à celles de J.-L. Nancy, dans Tombe de sommeil, Paris, Galilée, 2007, lequel conclut contrairement à Blumenberg à l'impossibilité d'une phénoménologie du sommeil.

${ }^{24}$ H. Blumenberg, Höhlenausgänge, Francfort-sur-le-Main, Suhrkamp, 1989 (désormais H), p. 15 sq.
} 
ne peut se vivre comme commençante. Même lors de l'éveil quotidien $\mathrm{du}$ sommeil, aucun instant n'est jamais le premier $»^{25}$. S'il est une limite entre veille et endormissement, entre sommeil et réveil, celle-ci n'est jamais appréhendable par un acte réflexif qui pourrait s'en saisir immédiatement ${ }^{26}$.

Les phénomènes limites ne peuvent être appréhendés qu'en deçà ou au-delà du seuil de leur actualité, et doivent être saisis comme discontinuités, voire approchés d'un point de vue extérieur, dans une phénoménologie qui renonce à l'idéal de l'immédiateté de la description. Celle-ci doit par conséquent se forger les moyens de dire et de donner à comprendre les phénomènes que la conscience expérimente comme son intermittence : le souvenir est ce dont je ne peux jamais être sûr qu'il se soit déroulé comme je m'en souviens, mais en quoi je me reconnais à travers le délai temporel. Les rêves sont ce dont, conformément à leur essence, on se réveille ${ }^{27}$, dans la troublante incertitude d'un contenu qui ne peut se saisir que comme évanouissant.

\section{La métaphore du flux de conscience.}

Ces différents phénomènes dont nous pourrions, grâce aux analyses de Blumenberg, davantage complexifier l'image générale, nous indiquent dans quelle mesure la conscience doit être comprise primordialement comme interrompue, transitoire, intermittente. Ouvrir cette perspective sur la conscience implique, pour Blumenberg, d'en passer par une analyse critique des métaphores par lesquelles Husserl décrit la conscience dans l'oblitération de ses phénomènes disruptifs. Parmi celles-ci, c'est évidemment celle du «flux », dont l'omniprésence et l'apparente évidence feraient presque oublier qu'elle est bien une métaphore, et que c'est par elle que la conception husserlienne de l'identité de la conscience gagne sa plausibilité, tout en omettant les difficultés qu'elle ne peut résoudre. Blumenberg développe des analyses métaphorologiques des textes husserliens ${ }^{28}$ dans lesquels on trouve en effet nombre de «flux», censés dire et saisir la continuité vivante de la conscience, l'identité à travers la multiplicité

\footnotetext{
${ }^{25} \mathrm{H}$, p. 10.

${ }^{26}$ Voir H, p. 15.

27 Voir ZSZ, p. 230.

${ }^{28}$ Voir QSE.
} 
de ses vécus, mais qui dans leurs contradictions, mettent au jour des lacunes de la phénoménologie husserlienne ${ }^{29}$.

Précisons tout de suite le statut de cette critique : jamais Blumenberg ne considère que les métaphores seraient inadaptées à la réflexion philosophique, ni qu'elles impliquent nécessairement des défauts d'argumentation; rien ne serait plus contraire à la perspective de cet auteur qui au contraire revalorise le rôle des métaphores et analyse leur efficace pour la pensée ${ }^{30}$. Elles peuvent en tout état de cause, et a fortiori dans le cas d'un philosophe comme Husserl pour qui le langage reste le moyen d'une description pure des phénomènes, indiquer les difficultés auxquelles se confronte son entreprise et offrir des ressources pour les résoudre ou les dépasser.

Chez Husserl, la métaphore du «Strom » (flux ou courant) devait remplacer les métaphores empiristes $\mathrm{du}$ "contenant» ou du «tableau », pour enfin faire valoir la conscience comme ensemble continu des vécus ${ }^{31}$ : le flux de conscience n'est pas autre chose que le "moi phénoménologique ${ }^{32}$. Mais les problèmes qui sont générés par cette métaphorique et en partie dissimulés par elle, sont nombreux. Quel est par exemple le statut de la réflexion? Où se situe le sujet lorsqu'il se tourne réflexivement vers ses propres vécus, dans des actes qui sont eux-mêmes des vécus ${ }^{33}$ ?

La métaphore du flux récuse le sensualisme empiriste et la conception atomistique de la conscience, sans en revenir à une représentation substantialiste. Mais elle présuppose une continuité, qui en fait ne peut être ni vécue ni pensée. Cette continuité est une fiction : «Nous le savons, car nous savons par expérience ce que signifie de veiller et de dormir, de s'endormir et de s'éveiller $»^{34}$.

Un des indices des impasses de la métaphorique du flux est l'introduction d'une autre métaphore, celle du "pôle » ${ }^{35}$, pour dire le sujet qui se maintient dans et par ce flux, faisant face au pôle de l'objectivité saisi par les vécus de conscience, et que Husserl nomme «monade». En elle doit se constituer l'identité de la conscience, qui rend ensuite possible l'identité du moi comme personne, avec des

\footnotetext{
${ }^{29}$ Voir QSE, p. 115 sq.

${ }^{30}$ Sur la «métaphorologie » de Blumenberg, voir principalement Paradigmes pour une métaphorologie, trad. D. Gammelin, Paris, Vrin, 2006, et la postface de J.-C. Monod à cet ouvrage.

${ }^{31}$ Critiquées dès les Recherches logiques.

${ }^{32}$ Voir QSE, p. 110.

${ }^{33}$ Voir QSE p. 112.

${ }^{34}$ Ibid., p. 163 . Nous traduisons.

${ }^{35}$ Notamment dans les Méditations cartésiennes, §31.
} 
habitus et une histoire personnelle. Or il y a une tension insoluble entre les métaphores du pôle et du flux, qui révèle la tension propre au projet de réduction de Husserl : la consistance du flux rend possible l'unité du pôle selon Husserl ${ }^{36}$, mais :

Si la conscience avait l'unité d'un flux, alors elle ne requerrait aucun principe égologique pour l'unification de ses contenus, bien plutôt ce sont eux qui engendreraient ce principe. D'un autre côté, la métaphore du flux devait perdre ses droits, dès lors que le Je s'était vu attribuer sa fonction de "pôle» à l'égard de chacun de ses contenus ${ }^{37}$.

L'introduction de la métaphore du pôle ne fait que traduire les apories de la phénoménologie transcendantale de Husserl, qui veut pouvoir garder les opérations d'identification du sujet égologique tout en le pensant dans la continuité de ses vécus.

L'ancien sujet, qui depuis les paralogismes de Kant ne pouvait plus être une substance ou avoir de substance, devait pourtant à nouveau accomplir leur tâche: constituer l'identité et la rapporter au flux du non-identique $e^{38}$.

L'attachement à l'identité de la conscience comme continuité ne peut que méconnaître les interruptions factuelles que connaît le sujet hu-

main et finit par le dédoubler, laissant la tâche de son unification à un sujet transcendantal qui se situe au-delà ou en deçà de sa vie empirique. Les ambitions de la phénoménologie l'obligent à partir d'un sujet réduit auquel viennent s'adjoindre ensuite les déterminations du moi, dans une tentative de le réincarner depuis sa structure abstraite.

\section{L'identité intermittente de la conscience}

L'analyse critique de Blumenberg débouche donc sur la nécessité d'une anthropologie phénoménologique qui considère la conscience depuis son intermittence. Ce faisant, Blumenberg court-circuite nombre des distinctions conceptuelles opératoires de Husserl : l'enjeu ne peut plus être de faire le partage entre empirique et transcendantal, nécessaire et contingent, passivité et activité.

Intentionnalité et indétermination

\footnotetext{
${ }^{36}$ Voir DH, p. 130.

${ }^{37}$ QSE, p. 110. Nous traduisons.

${ }^{38} \mathrm{Ibid}$., p. 129. Nous traduisons.
} 
Revenons donc sur le premier niveau des intermittences de la conscience; pour comprendre l'intentionnalité dans sa nature fragmentaire, Blumenberg développe une perspective anthropogénétique $^{39}$ qui reprend le questionnement génétique de la phénoménologie husserlienne tardive, mais en replaçant au cœur du propos la ou les questions anthropologiques. Il ne va pas de soi qu'il y ait quelque chose comme de la conscience, il n'est pas indispensable à l'autoconservation d'un organisme d'avoir la capacité de viser des objets. Ce que la conscience est et ce qu'elle peut doit donc être interrogé depuis ce qu'elle n'est pas ou plus et ne peut plus être, à savoir l'expé-

rience immédiate ou le rapport immédiat à ce qui l'entoure. Ainsi, la conscience doit être comprise comme le résultat d'une distance, d'une latence entre la face afférente et la face efférente de son rapport à $l^{\prime}$ environnement ${ }^{40}$. Selon Blumenberg, la conscience n'est en fait pas autre chose que cette distance même, la réponse à cet embarras presque funeste ${ }^{41}$.

Ce que nous nommons conscience n'est ni une adaptation (c'est-àdire le résultat de l'accumulation d'avantages du point de vue de la survie au cours de l'évolution), ni une forme inexplicable et qui ne devrait faire l'objet que d'une enquête transcendantale. Les ruptures et les interruptions auxquelles est soumise la conscience sont autant les conséquences de cette indétermination que les moyens dynamiques par lesquels elle s'en rend maître : la «valeur » de la conscience réside dans sa capacité à traiter l'hétérogénéité, par des procédures $\mathrm{d}^{\prime}$ « homogénéisation ${ }^{42}$ qui concernent les objets autant que les vécus. Dans les deux cas, elle ne doit pas être comprise comme une interjection mécanique, mais sur le modèle d'une dynamique de l'intermittence, qui éprouve la compatibilité des vécus entre eux ${ }^{43}:$ les opérations et les produits de la conscience doivent être compris comme des «médicaments » face aux « accidents » qu'elle rencontre dans la réalisation de son intentionnalité

[...] car si elle était parfaitement programmée pour la réalité, elle pourrait certes échapper à toute déception, mais elle serait également incapable d'expérience, et finalement serait démunie face à toute modification de la réalité. Les restitutions sont thésaurisées,

\footnotetext{
${ }^{39}$ Principalement dans DH, ZSZ, H et TL.

${ }^{40}$ Voir ZSZ, p. 135.

${ }^{41}$ Voir notamment $\mathrm{DH}$, « Le risque de l'existence et la prévention ».

${ }^{42}$ Voir QSE, p. 183.

${ }^{43}$ ZSZ, p. 43.
} 
Blumenberg ou les intermittences de la conscience

et c'est ainsi qu'est dégagée une marge de «liberté » pour l'imprévu $^{44}$.

${ }^{44}$ Ibid., p. 21. 
L'intentionnalité de la conscience est impensable sans cette économie seconde, ces procédures permettant de s'arranger de façon nécessairement provisoire avec l'indétermination de l'expérience. La conscience est à la fois distance et accès, latence et vigilance, distraction et attention, et doit être comprise dans son « élasticité » ${ }^{45}$, par laquelle seulement elle peut être un moyen au service de l'autoconservation de la vie humaine: "La conscience est aussi auto-constituante et auto-restituante que la vie elle-même ; elle est à son service et c'est cette fonctionnalité qui permet de la comprendre ${ }^{46}$. La conscience doit être comprise comme un arrangement, toujours temporaire et fragile, avec le dérangement qui reste sa condition de possibilité. Parmi ces «solutions de fortune » qui sont les conditions de possibilité subjective de l'expérience et que Blumenberg décrit à l'aide de métaphores thérapeutiques ou artisanales, il faut compter les médiations et les détours symboliques, conceptuels, logiques et métaphoriques $^{47}$ : «La manière dont l'homme se rapporte à la réalité (Wirklichkeitsbezug) est indirecte, circonstancielle, différée, sélective, et, surtout, "métaphorique" $\gg$.

Voilà ce qui devra faire l'objet de cette anthropologie phénoménologique, dont nous ne pouvons, dans le cadre de cette contribution, analyser toutes les dimensions et étapes. Pour ne mettre en avant qu'un concept, on pourrait remarquer à quel point l'attention devient centrale pour Blumenberg, elle est le paradigme de ces opérations secondes qui rendent possibles les opérations de la conscience qu'Husserl voulait considérer dans leur pureté essentielle. Husserl a bien consacré des réflexions au thème de l'attention, mais selon Blumenberg, il ne pouvait parvenir à lui accorder le rôle phénoménologique central qui lui revient ${ }^{49}$. Si la tendance intentionnelle de la conscience pouvait s'épanouir dans sa forme pure, alors chaque objet constituerait pour la conscience une tâche infinie. L'attention est le contrepoids nécessaire à la distraction, dans une dialectique qui rend possible ce que Husserl voulait considérer comme l'essence de la

\footnotetext{
${ }^{45}$ Voir $\mathrm{DH}$, «Le risque de l'existence et la prévention », où il est question de cette «élasticité » de la conscience.

${ }^{46} \mathrm{ZSZ}$, p. 20.

${ }^{47}$ Voir par exemple ZSZ p. 211 sqq., et H. Blumenberg, Arbeit am Mythos, Francfort-sur-le-Main, Suhrkamp, 1979, p. 28.

${ }^{48} \mathrm{H}$. Blumenberg, "Approche anthropologique d'une actualité de la rhétorique ", in L'imitation de la nature et autres essais esthétiques, trad. I. Kalinowski et M. de Launay, Paris, Hermann, 2010, p. 105. Traduction modifiée.

${ }^{49}$ Voir ZSZ, p. 192. Sur le thème de l'attention, voir E. Husserl, Hua 38, et Bernhard Waldenfels, "Wahrnehmung und Aufmerksamkeit beim frühen Husserl », Philosophische Rundschau, n 52, Tübingen, Mohr Siebeck, 2005, p. 302-310.
} 
conscience transcendantale : «L'attention résout la contradiction qui consiste en ceci qu'une conscience finie, conformément à l'essence de la conscience elle-même $[\ldots]$ a une tendance immanente à l'infini $\gg^{50}$.

\section{L'identité par intermittence}

Venons-en maintenant au second niveau des interruptions de la conscience, celui de son intermittence au sens strict. La perspective que propose Blumenberg permet de comprendre la tension produite par les phénomènes disruptifs comme une ressource: l'identité de la conscience ne se maintient pas malgré les interruptions, ou parce qu'elle réussirait à se rendre immune à celles-ci ; le soi et son identité se constituent seulement dans et par les réparations de ruptures ${ }^{51}$ et la connexion de vécus séparés dans le temps.

Peut-il y avoir des expériences qui brisent l'identité du sujet? Un kantien devrait affirmer qu'il ne saurait tout simplement $y$ avoir d'expérience rompant l'unité de la conscience, dans la mesure où elle anéantirait les conditions de possibilité de l'expérience elle-même, et représenterait donc une contradiction. Je tiens cette objection pour justifiée, mais considère pourtant d'une part qu'il est inévitable d'envisager la nécessité permanente de la conservation de l'unité de l'expérience comme ce vers quoi tendent les opérations de la conscience, d'autre part que le concept-limite de la donation de fait de l'identité - par exemple dans l'identité entre le Je qui se souvient et le Je dont il se souvient-est adéquat ${ }^{52}$.

Le vécu d'identité se constitue dans la réparation des interruptions. Prendre sur l'identité la perspective qu'offre son intermittence permet de comprendre dans quelle mesure elle se gagne paradoxalement elle-même au travers de phénomènes dont elle ne peut, à proprement parler, être le sujet et qui ne peuvent être, de façon immé-

diate, ses vécus.

\section{Les reprises de l'identité}

Dans un texte intitulé «In freier Variation: Identität », Blumenberg rappelle une anecdote racontée par Arthur Schnitzler, à propos d'un homme qui, chaque soir, s'envoyait à lui-même une lettre. Cette histoire tout d'abord seulement déroutante voire

\footnotetext{
${ }^{50}$ ZSZ, p. 199.

${ }^{51}$ Voir ZSZ, p. 35.

${ }^{52}$ Ibid., p. 82.
} 
fantasque traite, selon Blumenberg, de façon symbolique, d'un problème élémentaire de l'existence : une telle lettre serait le moyen de contourner extérieurement la nuit, cette « interruption fatale de la vie », elle offrirait, par le détour épistolaire, la figure d'une évidence toujours problématique : celle de la connexion identifiante à soi-même ${ }^{53}$.

Quand Blumenberg écrit ensuite que cet homme était un individu qui avait su " enfin » se montrer à la hauteur du danger que représente le sommeil, il ne faut pas éluder cette remarque comme si elle était un simple trait d'esprit paradoxal. Au-delà de l'asymétrie que l'on pourrait constater entre d'un côté le lourd problème philosophique de l'identité à soi, et de l'autre l'artifice dérisoire d'une lettre adressée à soi-même, elle vaut comme incitation à penser les médiations quotidiennes de la "correspondance » (ici au double sens du terme) avec soi-même. Le sommeil est une épreuve presque inadmissible pour le réalisme et la permanence de l'identité, quand bien même le défi qu'elle représente disparaîtrait sous les opérations et réparations qui la déconstruisent et qui assurent une forme de confiance spontanée quoique médiate dans la permanence de soi et du monde.

Peu importe ce que cet homme pouvait bien écrire dans sa relation épistolaire avec lui-même : ce n'est pas le contenu d'un message qui garantit l'identité, pas le souvenir du jour passé lui-même, mais plutôt l'efficace phatique par lequel il correspond avec lui-même.

On pourrait se demander si cet individu, le jour suivant, ne pourrait pas pour autant douter du fait qu'il est bien l'auteur de cette lettre lui provenant du jour précédant. Blumenberg pousse ici l'anecdote dans ses retranchements pour en extraire ce qu'elle peut nous dire du rapport à soi, qui reste hanté par la résurgence de doutes légers, certes vite évacués, mais troublants toutefois, de ceux qui nous viennent lorsque nous nous voyons obligés d'affirmer: «je ne me comprends plus moi-même $»^{54}$.

Une telle anecdote devrait appartenir à la variation phénoménologique, dès lors qu'elle cesse de récuser l'anthropologie, car comme nombre d'autres cas pathologiques - qu'ils soient réels ou fictifs - elle permet de mettre au jour les embarras de l'identité : "Nous vivons

\footnotetext{
${ }^{53}$ H. Blumenberg, Begriffe in Geschichten, Francfort-sur-le-Main, Suhrkamp, 1998 (désormais BG), p. 90.

${ }^{54}$ Ibid., p. 92.
} 
toujours entre ces deux extrêmes : le délitement et la réintégration » ${ }^{55}$. Non que nous expérimentions toujours réellement ces extrêmes, qui marquent simplement les bordures de toute la palette de l'entre-deux du rapport à soi. Voilà ce qui doit faire l'objet d'une phénoménologie de l'identité : ces «arts de la survie » quotidiens en relation avec ces « pannes» de la conscience ${ }^{56}$. Le souvenir, l'attente, l'éveil ne sont pas autre chose, avec pourtant tous les doutes et toutes les déceptions qu'ils impliquent, que des "formes de vécu de l'identité " ${ }^{57}$. L'identité n'est ni présence à soi transparente, ni conquête héroïque, elle s'obtient dans les opérations de réparation, de reprise de ses déchirures, et c'est ce qui signe l'ambivalence des phénomènes qui nous ont intéressé ici : "Le souvenir est toujours un manque d'authenticité de la conscience, sa défaillance à être entièrement elle-même. Mais tout de même aussi sa capacité à se reconstruire ${ }^{58}$.

On ne sera pas surpris, au vu des analyses de Blumenberg que nous avons présentées, que les descriptions les plus pertinentes de cette dynamique de l'intermittence soient à lire chez des écrivains plus que chez des phénoménologues, et notamment chez Proust que nous avions déjà évoqué :

Le début de la «Recherche" montre que nous ne pouvons pas avoir de commencement et que nous ne sommes pourtant pas en mesure $d^{\prime} y$ renoncer. Le paradoxe se révèle à celui qui souffre $\mathrm{d}^{\prime}$ insomnie et fait l'effort de se rappeler les moments où il parvenait à s'endormir et s'éveiller: ce que cela avait signifié $\mathrm{d}^{\prime}$ 'abandonner, de renoncer à soi et au monde environnant, s'abimer dans la perte de conscience [...] pour en émerger à nouveau et regagner le monde et soi $[\ldots]^{59}$.

Nous ne pouvons ici qu'esquisser les pistes sur lesquelles débouche cette anthropologie phénoménologique, et les modalités selon lesquelles elle se développe chez Blumenberg. Disons en tout cas qu'elle implique une phénoménologie de la corporéité, de l'intersubjectivité et des interactions sociales qui s'éloigne de celle de Husserl, et entre en dialogue ou résonance avec d'autres phénoménologues (MerleauPonty et Schütz notamment), écrivains et sociologues. Au début d'un texte intitulé « Realität ist das Selbe und doch nie das Gleiche, zu dem

\footnotetext{
${ }^{55}$ Ibid., p. 89.

${ }^{56}$ Voir LZWZ, p. 49 sq. Voir également ZSZ, p. 52.

${ }^{57}$ ZSZ, p. 142 sq.

${ }^{58}$ DH, p. 185.

${ }^{59} \mathrm{H}$, p. $14 s q$.
} 
man zurückkommt ${ }^{60}$, Blumenberg revient sur le début de l'œuvre de Proust et présente l'analyse du banal phénomène de la salutation, dont on peut considérer qu'il appartient aux opérations nécessairement intersubjectives par lesquelles la conscience correspond de nouveau avec elle-même.

J'émerge du sommeil, comme si je n'avais jamais existé. Il n'y a qu'un petit détail qui m'empêche d'être ce novice de l'existence: mon souvenir et ceux que les autres ont de moi. Il y a des circonstances, un contexte, des choses qui ne s'accommodent pas de l'idée que je pourrais faire mon apparition parmi eux pour la première fois. Je les connais, ils me connaissent. C'est pourquoi cela a un sens d'être salué par les autres tout comme je les salue, lorsque nous nous rencontrons le matin ou au cours de la journée. Aucune inti-

mité particulière n'est nécessaire, seulement une appartenance au sens le plus large du terme. La normalité du fait « d'avoir déjà été là » requiert le témoignage d'autrui pour un être qui peut dormir aussi profondément que l'homme, pour qui la jonction avec la vie préalable qu'il a vécue ne va pas de soi $^{61}$.

Rien de plus quotidien, rien de moins évident pour la conscience que de s'éveiller et de s'endormir. La pathologie de l'insomnie, affirme Blumenberg, correspondrait en fait bien mieux à la «normalité de l'existence » et au maintien de l'identité. À l'opposé, l'identité se réalise par des techniques qui ont un sens intersubjectifs. Les autres sont ceux qui m'empêchent d'être totalement autre chose que moi, qui produisent mon identité, dans une trivialité qui ne va pas de soi. Contrairement à ce qu'on pouvait penser, l'identité de la conscience à elle-même doit toujours être comprise comme « une forme de distance à soi » :

Ce que je veux montrer est l'application à moi-même de la réalisation de la négation qui consiste à penser du présent comme absent. Car cela seul signifie : former un concept de moi-même. $C^{\prime}$ est de là que naît, et $c^{\prime}$ est en cela que consiste la conscience de soi. À l'encontre de la littéralité, il s'agit d'une forme de distance de la conscience à elle-même ${ }^{62}$.

De la même façon, c'est l'intermittence de notre présence au monde qui devient condition de possibilité du réalisme de la conscience : le monde est ce dans quoi ou de quoi nous pouvons aussi être absent.

\footnotetext{
${ }^{60}$ L'allemand permet ici de distinguer entre deux sens de « mêmeté », contrairement au français.

${ }^{61} \mathrm{BG}$, p. 90.

${ }^{62}$ DH, p. 560.
} 
Le monde est ce qui peut être regagné : le monde commun par l'éveil, le monde de l'individu dans le souvenir, qui n'est rien d'autre que la persistance de l'identité contre les disruptions de la discontinuité, la perte, l'oubli63.

\section{Conclusion}

L'anthropologie phénoménologique de Blumenberg prend ses distances avec les ambitions principielles de la phénoménologie, pour thématiser les tensions constituantes de la conscience. L'identité de la conscience n'est pas seulement à décrire comme celle d'un être fini, mais aussi d'un être qui disparait ou s'absente à lui-même. Obtenir et maintenir son identité signifie être à même de se ressaisir dans les réparations qui connectent le présent et le passé, de "correspondre » avec soi-même (plus qu'à soi-même), de se faire reconnaître par les autres et se reconnaître par eux.

Blumenberg, en amont des procédures narratives par lesquelles le sujet peut se constituer au cours de sa biographie ${ }^{64}$, s'intéresse aux opérations que l'on pourrait qualifier de "proto-narratives » par lesquelles l'identité se constitue, et qui échappent nécessairement à une phénoménologie qui veut comprendre la conscience comme "flux». Si le «flux de conscience » peut avoir un sens, c'est plutôt au sens de la technique d'écriture qui donne à voir le dialogue intérieur de l'identité, les intermittences d'une conscience en déhiscence avec ellemême et qui se reprend, se reprise sans cesse. C'est le champ métaphorique de la couture qui ici nous servirait dans l'investigation de cette dynamique des intermittences. Dans Roland Barthes par Roland Barthes on lit, sous le titre « Patch-work» :

Me commenter? Quel ennui! Je n'avais d'autre solution que de me ré-écrire - de loin, de très loin - de maintenant : ajouter aux livres, aux thèmes, aux souvenirs, aux termes, une autre énonciation, sans que je sache jamais si c'est de mon passé ou de mon présent que je parle. Je jette ainsi sur l'œuvre écrite, sur le corps et le corpus passés, l'effleurant à peine, une sorte de patch-work, une couverture rapsodique faite de carreaux cousus. Loin d'approfondir, je reste à la surface, parce qu'il s'agit cette fois-ci de «moi » (du Moi) et que la profondeur appartient aux autres ${ }^{65}$.

\footnotetext{
${ }^{63}$ H, p. 16.

${ }^{64}$ Voir P. Ricœur, Soi-même comme un autre.

${ }^{65}$ R. Barthes, Par Roland Barthes, Paris, Seuil, 1975, p. 171.
} 
Ce qu'est, en elle-même, la conscience, l'essence de son identité, sont des problèmes auxquels la phénoménologie doit renoncer à vouloir répondre de façon absolue, pour laisser place à des descriptions sachant faire usage des ressources métaphoriques sans s'y piéger, puisant dans l'écriture littéraire et le travail de la fiction, se nourrissant des réflexions sur les pathologies du sujet. L'anthropologie phénoménologique de Blumenberg pose finalement la question de la diversité et du changement des formes que peut prendre la dialectique de l'intermittence de la conscience et de l'identité personnelle : les techniques de la correspondance de soi avec soi ont aussi une histoire. 